\title{
Cancer Stem Cells and the Tumor Microenvironment in Gastric Cancer
}

\author{
Ying Yang, Wen-Jian Meng ${ }^{*}$ and Zi-Qiang Wang \\ Department of Gastrointestinal Surgery, West China Hospital, Sichuan University, Chengdu, China
}

Gastric cancer (GC) remains one of the leading causes of cancer-related death worldwide. Cancer stem cells (CSCs) might be responsible for tumor initiation, relapse, metastasis and treatment resistance of GC. The tumor microenvironment (TME) comprises tumor cells, immune cells, stromal cells and other extracellular components, which plays a pivotal role in tumor progression and therapy resistance. The properties of CSCs are regulated by cells and extracellular matrix components of the TME in some unique manners. This review will summarize current literature regarding the effects of CSCs and TME on the progression and therapy resistance of GC, while emphasizing the potential for

OPEN ACCESS

Edited by:

Nathaniel Weygant,

Fujian University of Traditional Chinese

Medicine, China

Reviewed by:

Qianqian Song,

Wake Forest School of Medicine, United States

Ghmkin Hassan,

Hiroshima University, Japan

Dong-Joo (Ellen) Cheon,

Albany Medical College, United States

*Correspondence:

Wen-Jian Meng

mengwenijan@126.com

Specialty section:

This article was submitted to Gastrointestinal Cancers: Gastric

Esophageal Cancers,

a section of the journal

Frontiers in Oncology

Received: 28 October 2021 Accepted: 08 December 2021

Published: 03 January 2022

Citation

Yang $Y$, Meng W-J and Wang Z-Q (2022) Cancer Stem Cells and the Tumor Microenvironment in Gastric Cancer.

Front. Oncol. 11:803974.

doi: 10.3389/fonc.2021.803974 developing successful anti-tumor therapy based on targeting the TME and CSCs.

Keywords: gastric cancer, cancer stem cells (CSC), tumor microenvironment, mesenchymal stem cells, cancer associated fibroblasts (CAFs), tumor-associated macrophages (TAMs)

\section{INTRODUCTION}

Gastric cancer (GC) is the fifth most commonly diagnosed cancer and the fourth leading cause of cancer-associated mortality with an estimated more than one million new cases and 769,000 deaths worldwide in 2020 (1). Despite advancements in clinical treatment and technologies, the prognosis of GC remains poor, mainly due to relapse, metastasis and therapy resistance, and the median survival of patients with advanced GC is less than one year (2). Cancer stem cells (CSCs) are a minor subpopulation of uniquely tumorigenic cells exhibiting the capacity for self-renewal, unlimited proliferating and maintenance of a relatively dormant state. Since the first identification of CSCs in acute myeloid leukemia (3), increasing evidence suggests that CSCs may be responsible for tumor (including GC) progression, relapse, metastasis and therapy resistance (4-6).

Abbreviations: Ad5/35-DKK1, Adenovirus-mediated Dickkopf-1; ALDH, Aldehyde dehydrogenase; ALOX15, Arachidonate lipoxygenase 15; anti-PD-1, Anti-programmed cell death 1; anti-PD-L1, Anti-PD-1 ligand; ASIC1a, Acid-sensitive ion channel 1a; BM-MSCs, Bone marrow-derived MSCs; CAFs, Cancer associated fibroblasts; CagA, Cytotoxin-associated gene A; CaMKs, Calcium/calmodulin-dependent protein kinases; CSCs, Cancer stem cells; CTL, Cytotoxic T cells; DGCs, Diffuse-type gastric cancers; ECM, Extracellular matrix; EMT, Epithelial-mesenchymal transformation; EpCAM, Epithelial cellular adhesion molecule; FAK, Focal adhesion kinase; FAO, Fatty acid oxidation; FAP, Fibroblast activating protein; FDA, Food and Drug Administration; FGF, Fibroblast growth factor; GC, Gastric cancer; GCAFs, Gastric CAFs; GC-MSCs, GC-derived MSCs; GCSCs, Gastric CSCs; GEO, Gene Expression Omnibus; GNF, Gastric normal fibroblast; HH, Hedgehog; HIF, Hypoxia-inducible factor; HP, Helicobacter pylori; IL, Interleukin; MCP-1, Monocyte chemoattractant protein-1; MDR, Multi-drug resistance; MSCs, Mesenchymal stem cells; NK, Natural killer; PD-1, Programmed cell death 1; PDGF, Plateletderived growth factor; PD-L1, PD-1 ligand; RHBDF2, Rhomboid 5 Homolog 2; ROS, Reactive oxygen species; SCID, Severe combined immunodeficiency; SP, Side population; TAMs, Tumor-associated macrophages; TCGA, The Cancer Genome Atlas; TGF, Transforming growth factor; Th, Helper T cells; TILs, Tumor-infiltrating lymphocytes; TME, The tumor microenvironment; TNF, Tumor necrosis factor; Treg, Regulatory T cells; VCAM1, Vascular adhesion molecule 1; VEGF, Vascular endothelial growth factor. 
The cellular environment in which tumor cells reside is called the tumor microenvironment (TME), which consists of cellular and non-cellular components. It includes many types of stromal cells (fibroblasts, lymphocytes, macrophages, and endothelial cells), immune cells (such as $\mathrm{T}$ and $\mathrm{B}$ lymphocytes), and extracellular components (for instance: cytokines, growth factors, hormones and extracellular matrix), which surround tumor cells and are nourished by blood vessels around the tumor (Figure 1). The TME provides a suitable living environment for cancer cells to develop, escape from host immune surveillance and resist to anticancer drugs (7-10). With the continuous progress in the study of CSCs and TME, the microenvironment of CSC has gradually entered the vision of researchers. CSCs microenvironment (CSCs niche) is a special microenvironment for the survival of CSCs, which can regulate the characteristics of CSCs via cell-to-cell contact and secreted factors (11). Therefore, a precise and meticulous understanding of CSCs, TME and the relationship between them in GC will have a profound impact on the treatment of GC in the future. This review summarized current findings regarding the role of CSCs and TME in the progression of GC, which may facilitate the understanding of CSCs and TME of GC, as well as provide a potential therapeutic strategy based on targeting TME and CSCs for GC.

\section{IDENTIFICATION AND ISOLATION OF CSCs}

The study of CSCs may play a critical role in eradicating tumors and solving clinical problems such as tumor recurrence and

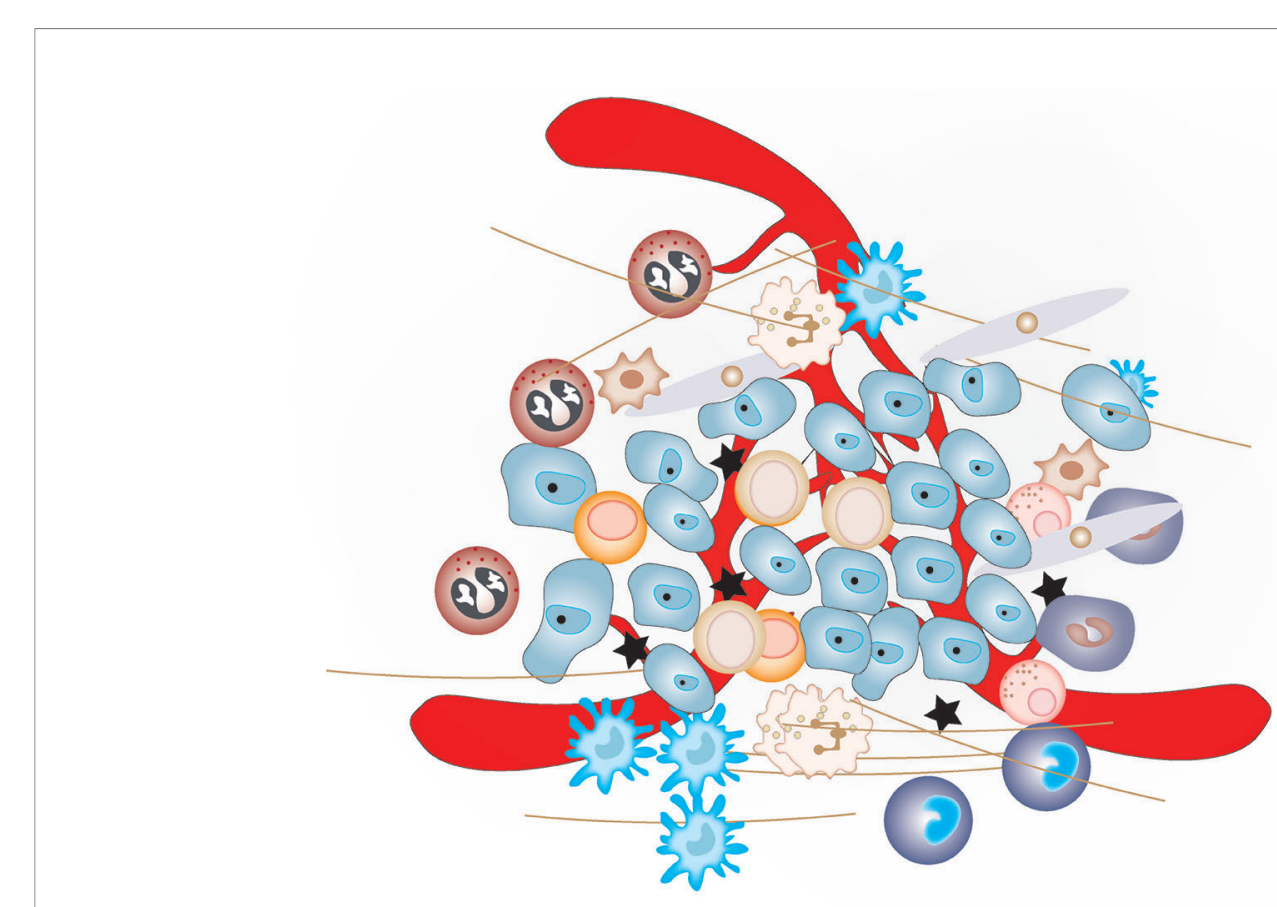

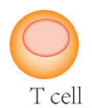
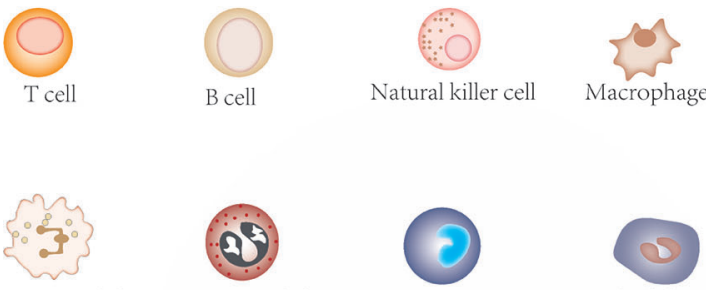

Neutrophil Eosinophil

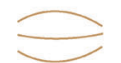

Extracellular matrix (ECM)
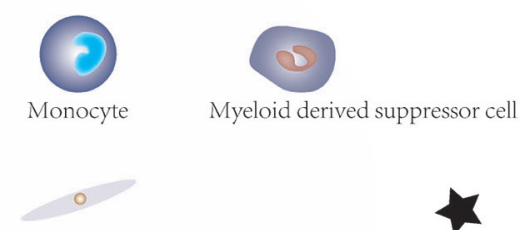

Cancer-Associated Fibroblast

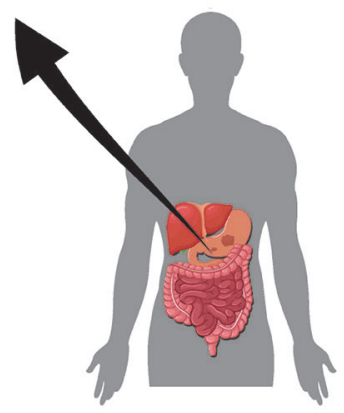

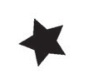

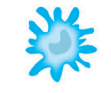

Dendritic cell

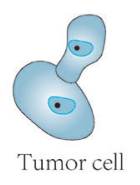

Cytokine/Chemokine

FIGURE 1 | The major constituents of the tumor microenvironment in gastric cancer. 
distant metastasis. However, the number of CSCs in cancer cells is extremely small (typically $<1 \%$ in solid tumors) (12). Therefore, the main difficulty in studying CSCs is how to identify and isolate them from a large number of tumor cells. At present, the most efficacious and commonly used method to isolate CSCs from a large number of tumor cells is by using specific cell surface markers of CSCs.

CSCs can be isolated and identified by combining specific cell surface markers of CSCs with corresponding monoclonal antibodies or fluorescein markers, and then applying some isolation techniques such as flow cytometry and magneticactivated cell sorting. Therefore, finding specific and effective cell surface markers of CSCs is critical for isolating and identifying CSCs. An increasing number of evidence has confirmed the existence of some specific cell surface markers in gastric CSCs (GCSCs). CD44 was the first confirmed potential GCSCs-specific cell surface marker. Takaishi et al. (13) found a considerable number of CD44 (+) cells in GC cell lines (MKN45, MKN-74, and NCI-N87), and these CD44 (+) cells showed spheroid colony formation in serum-free media in vitro. And when these cells were injected into the stomach and skin (around 30,000 cells per site) of severe combined immunodeficiency (SCID) mice, the significant tumorigenic ability in vivo was showed. However, only about $5 \%$ of the CD44 (+) cells were ultimately identified as true CSCs. In addition, CD44 is also widely expressed by nonmalignant tumors. Hence, it seems unlikely that a single cell surface marker of CD44 could detect all cells with the characteristics of GCSCs. Lau et al. (14) identified CD44v8-10 (the predominant CD44variant expressed in GC cells) as another potential cell surface marker of GCSCs. The results showed that the expression of CD44v8-10 was significantly upregulated in gastric tumor sites and that exogenous expression of CD44v8-10 contributed to tumor initiation in immunocompromised mice, possibly by improving oxidative stress defense. As a family of intracellular enzymes, aldehyde dehydrogenase (ALDH) enzymes are responsible for cell differentiation, detoxification and drug resistance through the oxidation of cellular aldehydes. Katsuno et al. (15) demonstrated that $\operatorname{ALDH1}(+)$ cells possess the characteristics of CSCs, accounting for about $5-8 \%$ of the human diffuse-type gastric carcinoma cells and displaying a higher tumorigenicity than ALDH1(-) cells. These findings indicate that ALDH1 is a potential specific cell surface marker for GCSCs. Numerous other molecules or proteins have also been suggested as potential cell surface markers for GCSCs. For instance, Jiang et al. (16). found that CD90(+) cells possessed an increased capacity of tumorigenicity in vivo compared with CD90(-) cells, and the expression level of CD90(+) cells was positively correlated with the tumorigenicity of GC cells in vivo. In addition, a combination therapy of trastuzumab with conventional chemotherapy is able to suppress tumor growth by reducing the proportion of CD90 $(+)$ cells. These findings suggest that CD90 may be a potential cell surface marker for GCSCs. Ohkuma et al. (17) have investigated the role of CSCs in gastric adenocarcinoma using MKN-1 cells, which showed that CD71 (-) cells were more tumorigenic than CD71 (+) cells in the gastric adenosquamous carcinoma model and that most CD71 $(-)$ cells were dormant (G1/G0 cell cycle phase) and resistant to 5 -FU. These characteristics of CD71 (-) cells were highly consistent with the characteristics of CSCs. Wenqi et al. (18) demonstrated that the epithelial cellular adhesion molecule (EpCAM) was overexpressed by gastric cancer cells and downregulation of EPCAM was able to inhibit tumor formation, reduce cell proliferation, and reduce the proportion of cells in a relatively dormant state. However, there are still some limitations and controversies about the use of these molecules as separate markers. Combining several molecular as cell surface markers to improve the ability of specifically identifying and isolating GCSCs has also been confirmed by some studies. For instance, CD44 was combined with other molecules as a specific cell surface marker for GCSCs, including CD24 (19), CD54 (20) and EpCAM (21). Table 1 summarizes the current specific cell surface markers for GCSCs.

In addition to the isolation of CSCs by cell surface markers, some characteristics of CSCs also have been applied to isolate and identify CSCs. In serum-free medium supplemented with growth factors, most non-CSCs cannot survive, while CSCs can survive and maintain their self-renewal characteristics. Therefore, serum-free medium containing growth factors can enrich and isolate CSCs, and $\mathrm{Li}$ et al. (23) used serum-free medium to enrich and isolate potential GCSCs. Side population (SP) cell isolation also can be applied to sort and enrich GCSCs by taking advantage of efflux characteristics of CSCs for Hoechst33342 (a nucleic acid dye) $(24,25)$. Traditional two-dimensional (2D) cell cultures do not mimic TME in vivo due to the lack of cell-extracellular matrix interactions. Animal models may also not adequately mimic the characteristics of human cancers. Therefore, the 3-dimensional (3D) culture

TABLE 1 | Cell surface markers of gastric cancer stem cells (GCSCs).

\begin{tabular}{|c|c|c|}
\hline GCSCs surface marker & Characteristic of stem cell & Reference \\
\hline CD44(+) & Tumorigenicity, self-renewal, multipotent differentiation, chemoresistance, colony-forming ability & (13) \\
\hline CD44v8-10 & Tumorigenicity, & $(14)$ \\
\hline ALDH & Chemoresistance, self-renewal, colony-forming ability, generate heterogeneity & $(15,22)$ \\
\hline CD90 & Tumorigenicity, self-renewal & (16) \\
\hline CD71 (-) & Tumorigenicity, self-renewal, relatively dormant state & (17) \\
\hline EpCAM & Tumorigenicity, relatively dormant state & (18) \\
\hline CD44(+)/CD24(+) & Tumorigenicity, self-renewal, multipotent differentiation & (19) \\
\hline CD44(+)/CD54(+) & Tumorigenicity, self-renewal & (20) \\
\hline EpCAM(+)/CD44(+) & Tumorigenicity, chemoresistance & (21) \\
\hline
\end{tabular}


system, which can better simulate in vivo cancer environment, has been widely used in cancer research. In addition, 3D culture systems can simulate the TME of CSCs by controlling the mechanical properties of materials and then effectively isolate CSCs. Recently, increasing studies have also used 3D culture systems to isolate and culture GCSCs (26-30).

With the continual development of single cell technologies, it is possible to identify CSCs from cancer cells. CSCs are usually difficult to isolate owing to their low abundance and similarity to other stem cells. Single-cell sequencing technologies are able to detect extremely trace amounts of nucleic acid sequences, which may assist in the identification and study of CSCs (31). For instance, Velten et al. (32) successfully identified leukemic stem cells from acute myeloid leukemia by clonal tracking from singlecell transcriptomics. Yang et al. (33) performed single-cell RNA sequencing of 59 cells from three bladder cancer samples, and finally found six key modifier genes (ETS1, GPRC5A, MKL1, PAWR, PITX2, and RGS9BP) in bladder CSCs that have never been reported. However, with the wide application of single-cell sequencing in the study of tumors, a large quantities of single-cell genomics data also makes it tricky to study. Therefore, Song et al. $(34,35)$ developed two research models of single-cell genomics data (single-cell Latent-variable Mode and Single-Cell Graph Convolutional Network) for better mining and understanding these data, which will aid in the understanding the complex mechanisms of cancer and CSCs. However, the high price of single-cell sequencing and the complexity of sample handling and operational procedures limit its application, and current studies identifying GCSCs by single-cell sequencing have not been reported.

\section{THE TME IN GC}

The TME favors the survival of tumor cells and provides an excellent shelter for them to escape host immune surveillance and resist anti-tumor drugs. Meanwhile, cancer cells in the TME can also affect and change their surrounding cells in an autocrine and a paracrine manner to maintain the TME required for the survival of cancer cells. This section will summarize the effects of the main components of TME on GC and the features of gastric TME.

\section{Cancer Associated Fibroblasts}

Cancer associated fibroblasts (CAFs) are the most abundant stromal cells in the TME, accounting for about 50\% of the total number of tumor tissue cells (36). CAFs can promote tumor development, proliferation, drug resistance, invasion and metastasis through direct contact or secretion of a variety of cytokines and metabolites in a paracrine manner, thereby affecting the prognosis of tumors. There are several controversies about the origins of CAFs, but an increasing number of evidence shows that CAFs originate from a variety of cells, such as bone marrow-derived cells, CSCs, epithelial cells through epithelialmesenchymal transformation (EMT) and normal fibroblasts. The diversity of origins of CAFs also contributes to the heterogeneity of CAFs (37-40) (Figure 2). CAFs not only provide physical support for epithelial cells in TME, but also are key factors for EMT of GC cells (41). EMT is a pathological process closely related to tumor invasion and metastasis. The main changes were that the cells changed from closely arranged epithelial cells to loosely structured interstitial cells, which weakened the adhesion between cells and increased the invasion and metastasis of the tumor. Angiogenesis is considered to be critical for tumor proliferation and metastasis, and vascular endothelial growth factor (VEGF) plays a crucial role in promoting angiogenesis. In GC cells, CAFS has been shown to promote angiogenesis by secreting FGF, IL-6, PDGF and VEGF and promote EMT by secreting transforming growth factor beta (TGF- $\beta$ ), FGF, TNF- $\alpha$, and IL-1 $\beta$, in turn leading to proliferation, invasion and metastasis of GC $(39,42-$ 49)(Figure 2).

CSCs have been shown to be capable of converting mesenchymal stem cells (MSCs) into CAFs in GC via exosome-mediated TGF- transfer and activation of TGF-/Smad pathways (50). In turn, CAFs are also able to maintain the stemlike properties of GC, promote the progression of GC and predict the prognosis of GC patients using the characteristics of CAFs. Hasegawa et al. (42) showed that CAFs may be able to maintain the stemness of sclerosing GC cells through TGF- $\beta$ signaling. And Spondin-2 secreted by CAFs in GC is positively correlated with peritoneal dissemination, tumor size and poor prognosis of GC (51). Ishimoto et al. (52) isolated CAFs and adjacent noncancer fibroblasts from resected specimens of 110 patients with diffuse-type GCs (DGCs) to investigated the characteristics and functions of CAFs in DGCs by analyzing the features of their genome and gene expression patterns. They found that DGCs cells cultured with CAFs were also more aggressive and invasive in vitro than those not cultured with CAFs. Further work using quantitative reverse PCR revealed that the expression of the Rhomboid 5 Homolog 2 (RHBDF2) gene associated with TGF$\beta 1$ activity was increased in DGC cells, and increased expression of RHBDF2 gene was observed after incubation of non-cancer fibroblasts with interleukin 1 alpha (IL- $1 \alpha$ ), IL- $\beta$ or TNF, secreted by DGCs. In view of the above findings, it was concluded that CAFs were able to activate TGF- $\beta 1$ signaling by increasing the expression of RHBDF2. And the activation of TGF- $\beta 1$ was demonstrated to increase the motility and invasiveness of GC cells. Fibroblast activating protein (FAP) is a member of the TME, Wen et al. (53) found that overexpression of FAP was negatively correlated with the survival rate of GC patients, and FAP combined with CAFs could promote the proliferation and invasiveness of GC cells and induce the development of chemoresistance of GC cells in vitro. In a xenograft model of GC, combined targeted inhibition of FAP and CAFs enhanced the antitumor immunity of immune checkpoint inhibitors. In addition, the characteristics of CAFs can also be used to predict the prognosis of GC patients and estimate the response of GC patients to clinical immunotherapy. Zheng et al. (54) constructed a 4-gene (COL8A1, SPOCK1, AEBP1, and TIMP2) prognostic CAFs model by analyzing mRNA expression and clinical information of GC samples 


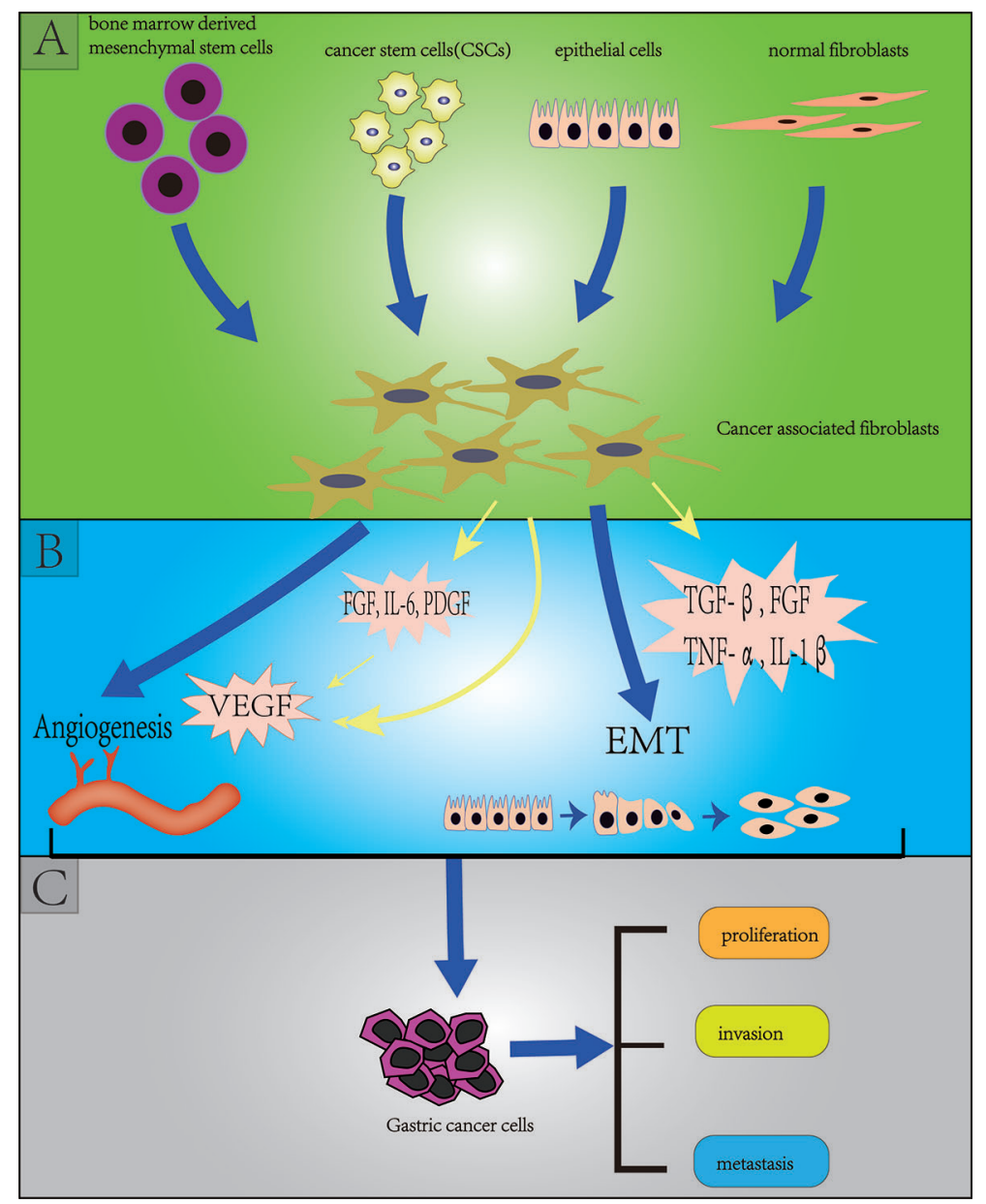

FIGURE 2 | Section (A) (green background) indicates the origins of cancer-associated fibroblasts (CAFs) including bone marrow derived mesenchymal stem cells, cancer stem cells, endothelial cells (through endothelial mesenchymal transition: EMT), normal fibroblasts; Section (B) (blue background) indicates the effect of CAFs on gastric cancer stem cells (GCSCs) including angiogenesis and EMT; Section (C) (grey background) indicates the effect of (GCSCs) on gastric cancer cells including proliferation, invasion and metastasis.

from GEO (Gene Expression Omnibus) and TCGA (Cancer Genome Atlas) databases. The results showed a positive correlation between CAFs risk score and stromal and CAFs infiltrations in GC, and patients in the high-CAF-risk group were less likely to respond to immunotherapy.

\section{Tumor-Associated Macrophages}

The most abundant immune cells in the TME are composed of macrophages or monocytes, which are called tumor-associated macrophages (TAMs). TAMs promote tumor progression by secreting a variety of factors, including growth factors, chemokines, cytokines, proteases and so on. Monocytes or macrophages are usually polarized into two main types: M1 and M2 (55). M1 macrophages enhance the function of T cells by releasing proinflammatory cytokines, such as TNF- $\alpha$, IL-1 and IL-12 and participate in type I helper $\mathrm{T}$ cell (Th) responses, which are crucial components involved in inflammatory responses and anti-tumor immunity (56). TAMs frequently exhibit an M2-like phenotype in the microenvironment of cancer, including GC, and express anti-inflammatory cytokines such as IL-10, TGF- $\beta$ and arginase (57). These antiinflammatory cytokines inhibit $\mathrm{T}$ cell-mediated anti-tumor immunity and provide tumors with an immunosuppressive microenvironment, allowing tumors to evade host immune surveillance and promote tumor growth and metastasis (58). In addition, Oishi et al. (59) demonstrated that intraperitoneal presentation of M2-polarized macrophages was able to inhibit $\mathrm{T}$ cell proliferation in vitro. In a mouse model of GC, TAMs were demonstrated to be able to secrete the proinflammatory factor TNF- $\alpha$, which contributes to the formation and development of GC by activating the Wnt signaling pathway $(60,61)$. Yamanaka et al. (62) showed that IL-1 $\beta$ secreted by TAMs of GC could increase the invasiveness of GC cells by activating NF- $\mathrm{KB}$ and expressing MMP-9. In addition, TAMs can also promote tumor angiogenesis and provides nutrition for tumor growth. Some studies have shown that the level of TAMs is closely associated 
with the number of blood vessels surrounding the GC cells. Ohta et al. (63) found that expression level of monocyte chemoattractant protein-1 (MCP-1) was significantly increased compared with negative tumors in human GC cell lines, and its expression level was closely related to the secretion of VEGF. And the counts of TAMs are positively correlated with the counts of vessel. They concluded that MCP-1 induced by human GC cells may promote angiogenesis of GC cells by recruiting and activating TAMs. Then, Kuroda et al. (64) further confirmed that MCP-1 also has a similar effect and mechanism (by activating and recruiting TAMs) in a mouse xenograft model of GC. In addition to promoting angiogenesis in GC through MCP-1, TAMs may also directly promote angiogenesis and lymphangiogenesis of GC possibly by enhancing the expression of VEGF and VEGF-C (65).

\section{Mesenchymal Stem Cells}

As an important part of the TME, MSCs play a key role in the process of tumor development, including tumor neovascularization, metastasis, maintenance of the stemness of CSCs and the formation of an immunosuppressive TME by activating signaling pathway and secreting a variety of regulatory factors (66). During the growth of GC, MSCs are recruited into the TME of GC, and they are able to alter the TME and promote tumor growth by secreting a variety of factors. GC-derived MSCs (GC-MSCs) were reported to enhance the proliferation, migration, and promotion of angiogenesis of GC cells by secreting considerable the proinflammatory cytokine interleukin-8 (IL-8) (67). IL-15 secreted by GC-MSCs enhances the stem-like properties of GC cells, induces EMT of GC cells which in turn promotes migration and metastasis of GC cells by upregulating Tregs (Regulatory $\mathrm{T}$ cells) ratio and programmed cell death protein-1 expression in CD4 + T Cell (68). Huang et al. (69) found that PDGF-DD secreted by GC-MSCs was capable of promoting the migration and proliferation of GC cells in vitro and in vivo by phosphorylating PDGF- $\beta$. Wang et al. (70) found that GC-MSCs were able to significantly promote the growth and migration of HGC-27 and increase microRNA-221 expression through paracrine secretion. In addition, inhibiting the expression of IL-8, PDGF-DD and microRNA-221 were all able to block its tumor-supporting role on GC cells. Recent studies have demonstrated that MSCs are crucial in the progression of GC. GC-MSCs can promote the growth of GC cells and the polarization of macrophages in the GC microenvironment to the M2 type by considerable secretion of IL- 6 and IL-8, and M2 type macrophages can promote GC metastasis by promoting the EMT of GC cells (71). The role of GC-MSCs in promoting GC metastasis and EMT of GC cells was also confirmed in another study (72). In TME, bone marrow-derived MSCs (BM-MSCs) were able to produce CXCL16 through Ror2-mediated signaling. While CXCL16 could induce expression of Ror1 in MKN45 cells, thereby promoting the progression of GC by activating the CXCR6-STAT3 signaling pathway (73). In addition, Wnt5aRor2 signaling in BM-MSCs has been shown to promote the proliferation of GC cells (74).

\section{Tumor-Infiltrating Lymphocytes}

Recently, the effect of tumor-infiltrating lymphocytes (TILs) on GC has also been reported. TILs refer to lymphocytes that leave the blood and enter the tumor, which are a major component of the TME, including CD8 + T cells, CD4 + T cells, B lymphocytes and natural killer $(\mathrm{NK})$ cells $(75)$. CD8 $+\mathrm{T}$ cells, also known as cytotoxic $\mathrm{T}$ cells (CTL), are recognized as the main anti-tumor immune effector cells. The subsets of CD $4+\mathrm{T}$ cells are represented by Th1, Th2 and Treg. Th1 cells, which secrete IL-2 and interferon, play a crucial role in activating and promoting the proliferation of CD8 $+\mathrm{T}$ cell and NK cell. Th2 enhances humoral immunity by secreting cytokines such as IL-4 and IL-6, which promote maturation and clonal proliferation of B cells. Treg cells can suppress the immune response in the host, including inhibiting the activation of $\mathrm{NK}$ cells and the cytotoxic function of CD8 $+\mathrm{T}$ cells (76). Kono et al. (77) confirmed that as a kind of Treg, the high expression of CD4 (+) CD25 high T cells was closely related to the worse prognosis of gastric and esophageal cancer. Interestingly, the level of CD4 (+) CD25 high T cells decreased significantly after patients with GC undergoing radical resection. Zhuang et al. (78) revealed that the overexpression of IL-22(+) CD4(+) T cells and Th22 cells were associated with tumor progression and predicted reduced overall survival. In addition, CD8 (+) T cells that produce IL-7 can promote the progression of GC cells by promoting chemotaxis of myeloid-derived suppressor cells (79).

\section{Features of Gastric TME}

Helicobacter pylori (HP) was confirmed to be associated with approximately $75 \%$ of GC events worldwide as a group 1 carcinogen $(80,81)$. HP infection is able to significantly affect and change the microenvironment of the stomach by inducing chronic inflammation of the stomach, while the inflammatory response will promote EMT of GC through a variety of mechanisms. Alternatively, HP cytotoxin-associated gene A (CagA) can change many signaling pathways of the host cell by inducing DNA damage and changing DNA methylation, and then induce the production of GC EMT. In addition, it also induces the generation of GC EMT by down-regulating the expression of E-cadherin and up-regulating the expression of vimentin and twist (82-84). CagA is also able to activated NFKB and STAT3 signals and increased the expression of SNAIL1 protein, which is closely related to CAFs activation and EMT in GC cells (85). Zhang et al. (86) found that HP infected gastric epithelial cells could activate and recruit MSCs and promote the conversion of MSCs into CAFs, which may promote the EMT of gastric epithelial cells. Krzysiek-Maczka et al. (87) found that HP strains are not only able to induce EMT of normal rat gastric epithelium cells, but also induce differentiation of rat normal gastric fibroblasts into CAFs.

An acidic microenvironment is a basic characteristic of the metabolic environment of tumor tissue. Tumor cells utilize glycolysis to create an acidic microenvironment conducive to invasion and metastasis (88-90). Current studies suggest that both glycolysis and oxidative phosphorylation may be the 
metabolic pathways of CSCs $(91,92)$, but it is undeniable that the acidic microenvironment favors the progression of malignant tumors and the emergence of CSCs (93). For most normal cells, living in an acidic microenvironment is harmful, while tumor cells can survive in the acidic environment and rely on the acidic microenvironment to maintain their ability to grow and proliferate rapidly (94). An acidic microenvironment and acidsensitive ion channel 1a (ASIC1a) were confirmed to promote the proliferation and migration of GC cells. Chen et al. (95) found that the expression of ASIC1a was significantly increased in GC tissues with postoperative metastasis compared with GC tissues without postoperative metastasis and non-tumor tissues. In addition, down-regulation of ASIC1a expression by silencing ASIC1a via shRNA was able to reduce the migration and invasion of GC cells. However, the regulatory mechanism of the acid-base microenvironment on tumor cell growth and metastasis is still unclear, and regulating the $\mathrm{pH}$ value of the
GC microenvironment may be an effective measurement to kill GC cells.

\section{INTERACTION BETWEEN CSCs AND TME}

With the continuous deepening of research in cancer, it has been slowly discovered that there is a complex dialogue between CSCs and the TME during tumor development. TME can not only affect the self-renewal ability of CSCs, but it may also induce the transformation of its surrounding non-tumor stem cells into CSCs (96). The TME surrounding CSCs can secrete cytokines such as hypoxia-inducible factor (HIF) and IL-1 3 , activate related signaling pathways, and participate in CSCs invasion and metastasis by inducing angiogenesis, induction of EMT and protecting CSCs from being attacked by the host's immune system. CSCs may also rely on their surrounding microenvironment to maintain their stem

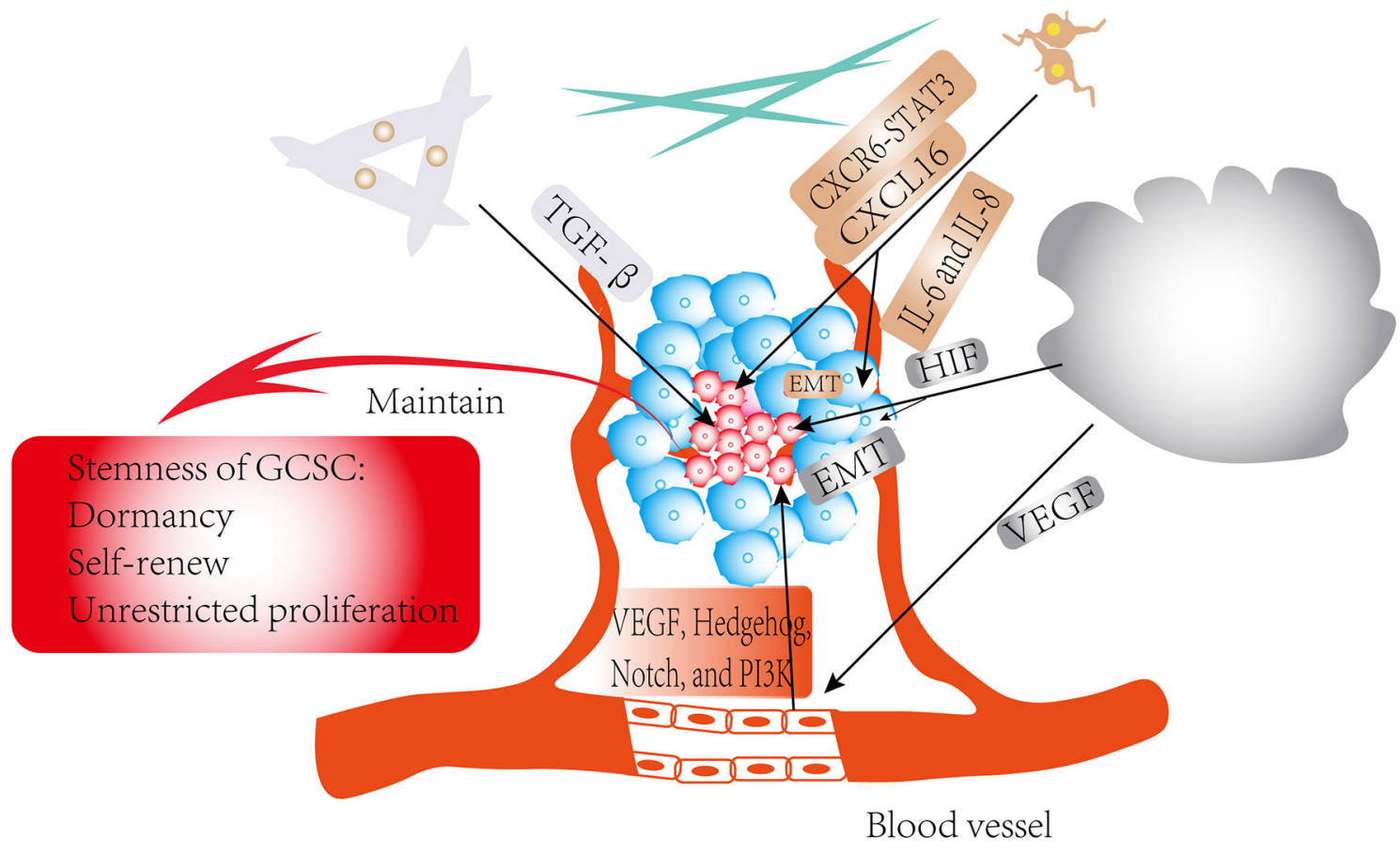

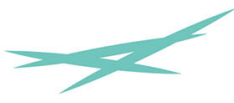

extracellular matrix

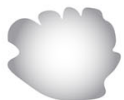

hypoxic microenvironment

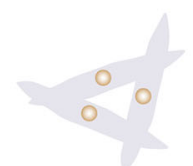

Cancer associated fibroblasts

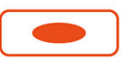

endothelial cells

Gastric cancer stem cells

Gastric cancer cells

FIGURE 3 | Regulatory role of TME in gastric cancer stem cells (GCSCs): The effects of members of the TME on GCSCs are shown by black arrows; the role of TME in maintaining the stemness of GCSCs is shown by red arrows. 
cell characteristics, such as self-renewal, dormancy and multilineage differentiation potential (Figure 3) (6, 97-101). Furthermore, the CSCs can also affect and modify the nature of the microenvironment. There is increasing evidence that CSCs are able to recruit and activate special types of cells such as MSCs to form a microenvironment suitable for the survival of CSCs, which is commonly known as the CSCs niche. The CSCs niche consists of stromal cells, immune cells, extracellular matrix (ECM), a vascular network and soluble factors (102). At present, there is little literature on exploring the interaction between CSCs and the TME in GC, and this paper only summarizes the existing literatures.

Recent studies have shown that CSCs reside in a vascular microenvironment that provides a suitable environment for the long-term growth of CSCs and maintenance of their stem-like properties. And vascular endothelial cells also play a crucial role in maintaining the stemness and self-renewal of CSCs. Some studies have shown that anti-vascular therapy (such as targeting VEGF) is effective in reducing the counts of CSCs and inhibiting tumor growth $(11,103)$. These studies demonstrate the critical role of perivascular microenvironment and vascular endothelial cells for the maintenance of the stemness of CSCs. Bevacizumab is a humanized monoclonal antibody that effectively prevents VEGF from binding to VEGFR-1, VEGFR-2, thereby inhibiting vascular endothelial cell proliferation and angiogenesis (104). Several studies have also demonstrated that targeted inhibition of the vascular microenvironment in GC can effectively inhibit the progression of GC. To explore the efficacy of bevacizumab in GC, a study in vitro suggested that the use of bevacizumab before chemotherapy can effectively improve the tumor control rate and reduce the tumor volume (105). In addition, it has been found that the combination of bevacizumab, docetaxel/oxaliplatin/5FU can increase the R0 resection rate of initially unresectable GC, indicating that bevacizumab is effective in the treatment of advanced gastric cancer (106). In addition, treatment with trastuzumab in combination with VEGF-Trap can effectively inhibit the development of HER2-overexpressing GC (107).

Although the perivascular microenvironment of CSCs is increasingly studied, recent studies have found that many CSCs also reside inside tumors that are far from blood vessels and in a hypoxic state, which is known as the hypoxic microenvironment of CSCs. It has been reported that hypoxia can maintain the stemness of CSCs and induce tumor cells to become biologically more aggressive such as invasion, metastasis and therapeutic resistance. These effects are mainly attributed to HIF $(108,109)$. However, the hypoxic microenvironment and perivascular microenvironment of CSCs are not hostile, and the hypoxic microenvironment is also able to promote angiogenesis. In addition, both endothelial cells and CSCs can produce VEGF to stimulate angiogenesis under a hypoxic environment. Hypoxia can protect CSCs from DNA-damaging agents including chemotherapy and radiation therapy, and also promote the survival and EMT of CSCs through reactive oxygen species (ROS)-activated stress pathway and TGF- $\beta$ signaling pathway. And hypoxia possess the ability of maintaining the self-renewal, tumorigenicity and the undifferentiated state of CSCs $(110,111)$. GCSCs microenvironment can also affect the characteristics of stem cells by regulating the expression of some transcription factors and tumor-related genes, and then affect the biological characteristics of tumor cells. For instance, Hasegawa et al. (42) confirmed that TGF- $\beta$ could blocks the effects of CAFs on GCSCs, reducing the number of SP cells in GCSCs and the level of surface marker expression of GCSCs. Maeda et al. (112) found that Wnt5a gene involved in stem cell niche may promote the invasive properties of GCSCs. It was reported that hypoxia is able to confer a stem cell-like phenotype on GC, enhancing drug resistance, radiation resistance, and EMT of GC cells (113-115). However, the specific mechanism of hypoxia affecting the characteristics of CSCs is still unclear and needs further research.

\section{ANTI-CANCER THERAPY TARGETING GC MICROENVIRONMENT AND GCSCs}

\section{Strategies for Targeting GC Microenvironment}

Most of the existing clinical drugs for GC treatment are aimed at the characteristics of rapid proliferation of tumor cells, such as cytotoxic chemotherapeutic drugs. Recently, with the in-depth understanding of the role and mechanism of TME in tumor progression, great progress has also been made in the study of targeted TME therapy for tumors including GC. Here, we will show some strategies based on targeting the GC microenvironment for the treatment of GC.

Immune checkpoints are pathways that inhibit the immune system through interactions between ligand and receptor and can regulate the immune system to avoid damage to normal cells and tissues. Cancer cells, on the other hand, evade the surveillance and killing of the immune system by using immune checkpoints, leading to the continuous progression of cancer. Recently, anti-programmed cell death 1 (anti-PD-1) and anti-PD-1 ligand (anti-PD-L1) antibodies with good anti-tumor effect have gradually attracted much attention (116). Briefly, PD-1 is a transmembrane protein on the surface of T cells, and T cells will not kill cancer cells when PD-1 binds to PD-L1 expressed on the surface of cancer cells. Anti-PD-1 antibody can bind to PD-1 expressed on $\mathrm{T}$ cells, while anti-PD-L1 antibody can bind to PD-L1 expressed on cancer cells, thus preventing PD-1 expressed on T cells from binding to PD-L1 expressed on tumor cells, allowing $\mathrm{T}$ cells to kill cancer cells (117). For instance, pembrolizumab (anti-PD-1 antibody) has been shown a significant survival benefit in the treatment of GC in many studies and has been approved by the Food and Drug Administration (FDA) as a third-line treatment for PD-L1-positive GC patients as well as patients with unresectable or metastatic, highly microsatellite instability or mismatch repair-deficient (118).

CAFs have already been recognized to play a key role in promoting GC progression. Therefore, drugs that inhibit the function of CAFs may potentially prevent the progression of GC. Wang et al. (119) cultured gastric CAFs (GCAFs) and gastric normal fibroblast (GNF) with BGC-823 human GC cells, respectively. The results of this study showed that GCAFs 
could significantly promote the proliferation, migration and invasion of BGC-823 cells by down-regulating microRNA-214 and up-regulating microRNA-301a compared with GNFs. And astragaloside IV, a main component of nontoxic Chinese herb, can inhibit the proliferation and invasion of GC by inhibiting the pathological function of CAFs through regulation of microRNA expression. Ferroptosis is a form of regulatory cell necrosis induced by lipid peroxidation (lipid-ROS), iron and reactive oxygen species. Zhang et al. (120) demonstrated that exosomal microRNA-522 secreted by CAFs was able to inhibit ferroptosis in GC cells by inhibiting arachidonate lipoxygenase 15 and reducing lipid-ROS production. In addition, cisplatin and paclitaxel were also demonstrated to be able to increase the secretion of microRNA-522 from CAFs by activating the USP7/ hnRNPAlaxis, resulting in decreased chemosensitivity of GC cells. And in another study, Uchihara et al. (121) showed Annexin A6 in extracellular vesicles (EV) from CAFs induced drug resistance of GC by activation of $\beta 1$ integrin-focal adhesion kinase (FAK)-YAP. They also revealed that inhibition of FAK or YAP was able to effectively attenuate drug resistance of GC in a mouse model of peritoneal metastasis. Shen et al. (122) showed $\mathrm{HP}$ infection can increase expression of vascular adhesion molecule 1 (VCAM1) in CAFs of GC by activating the JAK/ STAT1 signaling pathway, and the expression level of VCAM1 is positively correlated with the progression of GC and the poor prognosis of patients with GC. In addition, the interaction between CAFs-derived VCAM1 and integrin $\alpha v \beta 1 / 5$ can promote the invasiveness of GC in vivo and in vitro. These findings facilitate us to further understand the mechanism of how CAFs promote GC progression and drug resistance, and provide potential targeted therapeutic strategies for GC treatment and overcoming GC drug resistance.

As an essential component of TME, TAMs are considered to be closely associated with the progression, metastasis and drug resistance of GC. Among TAMs, those of the M2 type are responsible for inhibiting $\mathrm{T}$ cell function and promoting tumor growth. Therefore, several potential therapeutic strategies have been proposed to work on eradicating M2 TAMs or converting M2 TAMs into M1 TAMs. Miao et al. (123) performed an immunohistochemical analysis of STING expression in 200 pairs of GC cells and its surrounding normal tissues and detected the effects of STING on cancer cell apoptosis and $\mathrm{T}$ cell differentiation by flowcytometry. They also verified the results in a spontaneous GC model of p53+/- mice and cell line-based xenografts. The results of this study suggest that down-regulation of STING expression is able to promote TAMs polarizing into the M1 as well as induce apoptosis in GC cells through the IL6RJAK-IL24 pathway. Zheng et al. (124) found that M2-type TAMs were able to promote cisplatin resistance in CG cells. Further analysis using the microRNA profiles assay confirmed that exosomal microRNA-21 derived from M2-type TAMs conferred cisplatin resistance in GC cells. Wang et al. (125) confirmed in vitro that the expression levels of Legumain in TAMs were positively correlated with the proliferation and angiogenesis of GC. Further experiments in vivo also confirmed that GC cells injected with Legumain-knockdown TAMs showed slower growth and less angiogenesis compared with GC cells injected with TAMs. These studies showed that targeting exosomes associated with TAMs may be a promising new therapeutic strategy for the therapy of GC and overcoming drug resistance of GC.

Another important component in TME, MSCs plays a key role in the progression of GC and may be a promising therapeutic target for GC. Accumulating evidence indicates that MSCs contribute to progression and chemotherapy resistance of GC by secreting soluble molecules and regulating various signaling pathways. GC-MSCs have been confirmed to promote immune escape by secreting IL- 8 and can induce the expression of PD-L1 in GC cells. Sun et al. (126) further confirmed that GC-MSCs were able to enhance the stemness and self-renewal of GC cells through PD-L1, leading to chemoresistance of GC. It has also been confirmed that MSCs can promote the stemness and chemoresistance of GC cells both in vivo and in vitro through fatty acid oxidation (FAO). And FAO inhibitors have been demonstrated to reduce MSCs induced resistance to FOLFOX chemotherapy regimens in GC cells. These results of this study suggested that FAO was a key factor in MSC-induced stemness and chemoresistance of GC cell and inhibitors targeting FAO combined with conventional chemotherapy regimens may be a promising therapeutic strategy to overcome GC chemoresistance (127). In addition, exosomes secreted by MSC are responsible for 5-FU resistance in GC cells both in vivo and in vitro. Ji et al. (128) revealed that MSC-derived exosomes can prevent 5-FU -induced apoptosis of GC cells and increase the expression of multi-drug resistance (MDR)-associated proteins, such as MDR by activating the calcium/calmodulin-dependent protein kinases (CaM-Ks) and Raf/MEK/ERK signaling pathway. And inhibition of CaM-Ks/ Raf/MEK/ERK signaling pathway is also able to inhibit GC chemoresistance induced by MSC-exosomes. These findings suggest that targeting MSCs-related soluble molecules and signaling pathways combined with conventional chemotherapy may provide a promising new strategy to overcome the resistance of GC cells to conventional chemotherapy.

At present, surgical resection and chemoradiotherapy are the main therapeutic strategies for the treatment of GC, and molecular targeted drugs are an emerging therapeutic strategy for the treatment of GC. Increasing studies have shown that targeting key signaling pathways and molecules of the TME in GC may be a new promising therapeutic strategy for the treatment of GC. While knowledge is emerging regarding preclinical studies of the GC microenvironment, data from clinical studies on targeting the GC microenvironment in the therapy of GC is still limited. Thus, there is still a long way to go before the therapy of targeting the GC microenvironment can be applied in the treatment of GC. The underlying specific mechanisms involved in TME affecting the progression, recurrence, metastasis and drug resistance of GC remain poorly understood and further studies are still warranted.

\section{Strategies for Targeting GCSCs}

GCSCs are considered to be responsible for the development, recurrence, metastasis and drug resistance of GC. Therefore, therapeutic strategies for the targeted elimination of CSCs are 
considered to be one of the promising approaches for the treatment of GC. However, there are methodological dilemmas in the current therapeutic strategy for targeting CSCs, including the lack of detection methods to specifically identify and isolate CSCs from tumor cells. This paper summarizes the current promising strategies for targeting GCSCs.

There are some specific surface markers in the surface of GCSCs. Targeting these specific surface markers is an important way to kill GCSCs and improve the prognosis of GC. Many investigators have searched for specific surface markers of GCSCs for a long time, and CD44 has been confirmed in some studies as a specific surface marker of GCSCs. Yao et al. (129) developed a gastric CSCsspecifically targeting drug delivery system (SAL-SWNT-CHI-HA complexes) that can inhibit the self-renewal ability of CD44 (+) cells in serum-free medium, which in turn reduces the formation of GCSCs. Through the eradication of GCSCs, it can effectively eradicate GC cells and block the migration and invasion of GC cells. Liang et al. (130) developed a nanoprobe against CD44v6 (a surface marker of GCSCs), which specifically targets GCSCs. In orthotopic and subcutaneous xenograft models of GC, this nanoprobe actively targets the vascular system of GC and significantly inhibits the growth of GC at 4 hours post-injection. In addition, aberrant activation of signaling pathways is present in GCSCs, and a therapeutic strategy for targeting key signaling pathways in GCSCs appears theoretically feasible in reducing GCSCs and improving GC patient prognosis. GSI, a $\gamma$-secretase inhibitor IX, was reported to be capable of inhibiting the proliferation, migration, invasion, and tumor sphere formation of CD44 (+) GCSCs by inhibiting the Notch signaling pathway (131). Feng et al. (132) found that in a GCSCs model, pantoprazole was able to increase the therapeutic sensitivity of GC to 5-fluorouracil, decrease the capacity of generating tumor spheres and the expression levels of GCSCs markers such as CD44, CD24, and Lgr5 by inhibiting the EMT/ $\beta$-catenin pathways. In a study (133), CD44 (+) GC cells were shown to be resistant to 5-FU and cisplatin chemotherapy. In this study, Hedgehog $(\mathrm{HH})$ signaling played an important role in maintaining the stem-like properties of CD44 (+) GC cells, and inhibition of $\mathrm{HH}$ could increase the sensitivity of CD44 (+) GC cells to chemotherapy. Mao et al. (134) found that activation of Wnt signaling can promote the self-renewal and proliferation of GCSCs, and salinomycin can inhibit the growth of GC by inhibiting Wnt signaling in GCSCs. The chimeric 5/35 adenovirus-mediated Dickkopf-1(Ad5/35-DKK1) that could effectively attenuate Wnt signaling of GCSCs was developed by Wang et al. (135). And in preclinical experiments, Ad5/35-DKK1

\section{REFERENCES}

1. Sung H, Ferlay J, Siegel RL, Laversanne M, Soerjomataram I, Jemal A, et al. Global Cancer Statistics 2020: GLOBOCAN Estimates of Incidence and Mortality Worldwide for 36 Cancers in 185 Countries. CA Cancer J Clin (2021) 71(3):209-49. doi: 10.3322/caac.21660. 10.3322/caac.21660.

2. Smyth EC, Nilsson M, Grabsch HI, van Grieken NC, Lordick F. Gastric Cancer. Lancet (2020) 396:635-48. doi: 10.1016/S0140-6736(20)31288-5

3. Bonnet D, Dick JE. Human Acute Myeloid Leukemia Is Organized as a Hierarchy That Originates From a Primitive Hematopoietic Cell. Nat Med (1997) 3:730-7. doi: 10.1038/nm0797-730 was demonstrated to be able to inhibit the invasion of CD44 (+) GC cells. Although research on targeting GCSCs is accumulating, more studies, especially clinically relevant studies, are needed to demonstrate the clinical significance of therapeutic strategy for targeting CSCs.

\section{CONCLUSION AND FUTURE PERSPECTIVES}

The TME is a complex biological system composed of a variety of cells, extracellular matrix and biological molecules. It is closely related to tumorigenesis, invasion, metastasis, and immune evasion of GC cells by secreting a variety of factors and regulating signaling pathways. CSCs are a small population of tumor cells with stemlike characteristics, which also play a crucial role in the progression, metastasis and drug resistance of GC. On the one hand, TME and CSCs may synergistically contribute the progression of GC. On the other hand, TME and CSCs may mutually promote each other. And future studies to investigate the relationship among them will provide a new idea for the cancer progression and novel therapeutic targets in GC. Because various current targeted therapeutic strategies for GC are mainly to kill non-CSCs, and the residual CSCs of GC after current therapy are implicated in tumor recurrence and metastasis. Consequently, the development of therapy targeting CSCs is warranted for the effective treatment of GC. In addition, studies focusing on targeting TME in the progression and drug resistance of GC have also confirmed that TME may provide new targets for the treatment of GC. In the future, further study on the microenvironment of CSCs in GC should be carried out to clarify the different components and functions of the microenvironment of CSCs in GC. Which will be helpful to understand the mechanism underlying the pathogenesis of GC, develop new therapy to kill CSCs in GC and change GC microenvironment, leading to promote the clinical treatment of GC.

\section{AUTHOR CONTRIBUTIONS}

Literature review, data analysis, and manuscript preparation were performed by YY. W-JM and Z-QW contributed for the study conception, design, and revision. All authors contributed to the article and approved the submitted version.

4. Zhang L, Guo X, Zhang D, Fan Y, Qin L, Dong S, et al. Upregulated miR-132 in Lgr5 Gastric Cancer Stem Cell-Like Cells Contributes to CisplatinResistance via SIRT1/CREB/ABCG2 Signaling Pathway. Mol Carcinog (2017) 56:2022-34. doi: 10.1002/mc.22656

5. Shibue T, Weinberg RA. EMT, CSCs, and Drug Resistance: The Mechanistic Link and Clinical Implications. Nat Rev Clin Oncol (2017) 14:611-29. doi: $10.1038 /$ nrclinonc.2017.44

6. Batlle E, Clevers H. Cancer Stem Cells Revisited. Nat Med (2017) 23:112434. doi: $10.1038 / \mathrm{nm} .4409$

7. Cai W-Y, Dong Z-N, Fu X-T, Lin L-Y, Wang L, Ye G-D, et al. Identification of a Tumor Microenvironment-Relevant Gene Set-Based Prognostic 
Signature and Related Therapy Targets in Gastric Cancer. Theranostics (2020) 10:8633-47. doi: 10.7150/thno.47938

8. Choi H, Na KJ. Integrative Analysis of Imaging and Transcriptomic Data of the Immune Landscape Associated With Tumor Metabolism in Lung Adenocarcinoma: Clinical and Prognostic Implications. Theranostics (2018) 8:1956-65. doi: 10.7150/thno.23767

9. Wang M, Zhao J, Zhang L, Wei F, Lian Y, Wu Y, et al. Role of Tumor Microenvironment in Tumorigenesis. J Cancer (2017) 8:761-73. doi: $10.7150 /$ jca. 17648

10. Mashukov A, Shapochka D, Seleznov O, Kobyliak N, Falalyeyeva T, Kirkilevsky $S$, et al. Histological Differentiation Impacts the Tumor Immune Microenvironment in Gastric Carcinoma: Relation to the Immune Cycle. World J Gastroenterol (2021) 27:5259-71. doi: 10.3748/wjg.v27.i31.5259

11. Plaks V, Kong N, Werb Z. The Cancer Stem Cell Niche: How Essential Is the Niche in Regulating Stemness of Tumor Cells? Cell Stem Cell (2015) 16:22538. doi: 10.1016/j.stem.2015.02.015

12. Ishizawa K, Rasheed ZA, Karisch R, Wang Q, Kowalski J, Susky E, et al. Tumor-Initiating Cells Are Rare in Many Human Tumors. Cell Stem Cell (2010) 7:279-82. doi: 10.1016/j.stem.2010.08.009

13. Takaishi S, Okumura T, Tu S, Wang SSW, Shibata W, Vigneshwaran R, et al. Identification of Gastric Cancer Stem Cells Using the Cell Surface Marker CD44. Stem Cells (2009) 27:1006-20. doi: 10.1002/stem.30

14. Lau WM, Teng E, Chong HS, Lopez KAP, Tay AYL, Salto-Tellez M, et al. CD44v8-10 Is a Cancer-Specific Marker for Gastric Cancer Stem Cells. Cancer Res (2014) 74:2630-41. doi: 10.1158/0008-5472.CAN-13-2309

15. Katsuno Y, Ehata S, Yashiro M, Yanagihara K, Hirakawa K, Miyazono K. Coordinated Expression of REG4 and Aldehyde Dehydrogenase 1 Regulating Tumourigenic Capacity of Diffuse-Type Gastric CarcinomaInitiating Cells Is Inhibited by TGF- $\beta$. J Pathol (2012) 228:391-404. doi: $10.1002 /$ path. 4020

16. Jiang J, Zhang Y, Chuai S, Wang Z, Zheng D, Xu F, et al. Trastuzumab (Herceptin) Targets Gastric Cancer Stem Cells Characterized by CD90 Phenotype. Oncogene (2012) 31:671-82. doi: 10.1038/onc.2011.282

17. Ohkuma M, Haraguchi N, Ishii H, Mimori K, Tanaka F, Kim HM, et al. Absence of CD71 Transferrin Receptor Characterizes Human Gastric Adenosquamous Carcinoma Stem Cells. Ann Surg Oncol (2012) 19:135764. doi: 10.1245/s10434-011-1739-7

18. Wenqi D, Li W, Shanshan C, Bei C, Yafei Z, Feihu B, et al. EpCAM Is Overexpressed in Gastric Cancer and Its Downregulation Suppresses Proliferation of Gastric Cancer. J Cancer Res Clin Oncol (2009) 135:127785. doi: 10.1007/s00432-009-0569-5

19. Zhang C, Li C, He F, Cai Y, Yang H. Identification of CD44+CD24+ Gastric Cancer Stem Cells. J Cancer Res Clin Oncol (2011) 137:1679-86. doi: $10.1007 / \mathrm{s} 00432-011-1038-5$

20. Chen T, Yang K, Yu J, Meng W, Yuan D, Bi F, et al. Identification and Expansion of Cancer Stem Cells in Tumor Tissues and Peripheral Blood Derived From Gastric Adenocarcinoma Patients. Cell Res (2012) 22:248-58. doi: 10.1038/cr.2011.109

21. Han M-E, Jeon T-Y, Hwang S-H, Lee Y-S, Kim H-J, Shim H-E, et al. Cancer Spheres From Gastric Cancer Patients Provide an Ideal Model System for Cancer Stem Cell Research. Cell Mol Life Sci CMLS (2011) 68:3589-605. doi: 10.1007/s00018-011-0672-z

22. Nishikawa S, Konno M, Hamabe A, Hasegawa S, Kano Y, Ohta K, et al. Aldehyde Dehydrogenase High Gastric Cancer Stem Cells Are Resistant to Chemotherapy. Int J Oncol (2013) 42:1437-42. doi: 10.3892/ijo.2013.1837

23. Li G, Su Q, Liu H, Wang D, Zhang W, Lu Z, et al. Frizzled7 Promotes Epithelial-To-Mesenchymal Transition and Stemness Via Activating Canonical Wnt/B-Catenin Pathway in Gastric Cancer. Int J Biol Sci (2018) 14:280-93. doi: 10.7150/ijbs.23756

24. Wolmarans E, Nel S, Durandt C, Mellet J, Pepper MS. Side Population: Its Use in the Study of Cellular Heterogeneity and as a Potential Enrichment Tool for Rare Cell Populations. Stem Cells Int (2018) 2018:2472137. doi: 10.1155/2018/2472137

25. Haraguchi N, Utsunomiya T, Inoue H, Tanaka F, Mimori K, Barnard GF, et al. Characterization of a Side Population of Cancer Cells From Human Gastrointestinal System. Stem Cells (2006) 24:506-13. doi: 10.1634/ stemcells.2005-0282
26. Courtois S, Durán RV, Giraud J, Sifré E, Izotte J, Mégraud F, et al. Metformin Targets Gastric Cancer Stem Cells. Eur J Cancer (Oxford Engl 1990) (2017) 84:193-201. doi: 10.1016/j.ejca.2017.07.020

27. Chen S, Chen C, Hu Y, Zhu C, Luo X, Wang L, et al. Three-Dimensional Culture for Drug Responses of Patient-Derived Gastric Cancer Tissue. Front Oncol (2020) 10:614096. doi: 10.3389/fonc.2020.614096

28. Magalhães L, Quintana LG, Lopes DCF, Vidal AF, Pereira AL, D'Araujo Pinto LC, et al. APC Gene Is Modulated by hsa-miR-135b-5p in Both Diffuse and Intestinal Gastric Cancer Subtypes. BMC Cancer (2018) 18:1055. doi: 10.1186/s12885-018-4980-7

29. Kim D-H, Lee S, Kang HG, Park H-W, Lee H-W, Kim D, et al. Synergistic Antitumor Activity of a DLL4/VEGF Bispecific Therapeutic Antibody in Combination With Irinotecan in Gastric Cancer. BMB Rep (2020) 53:533-8. doi: 10.5483/BMBRep.2020.53.10.103

30. Takeuchi A, Yokoyama S, Nakamori M, Nakamura M, Ojima T, Yamaguchi $\mathrm{S}$, et al. Loss of CEACAM1 Is Associated With Poor Prognosis and Peritoneal Dissemination of Patients With Gastric Cancer. Sci Rep (2019) 9:12702. doi: 10.1038/s41598-019-49230-w

31. Chen T, Li J, Jia Y, Wang J, Sang R, Zhang Y, et al. Single-Cell Sequencing in the Field of Stem Cells. Curr Genomics (2020) 21:576-84. doi: 10.2174/ 1389202921999200624154445

32. Velten L, Story BA, Hernández-Malmierca P, Raffel S, Leonce DR, Milbank J, et al. Identification of Leukemic and Pre-Leukemic Stem Cells by Clonal Tracking From Single-Cell Transcriptomics. Nat Commun (2021) 12:1366. doi: 10.1038/s41467-021-21650-1

33. Yang Z, Li C, Fan Z, Liu H, Zhang X, Cai Z, et al. Single-Cell Sequencing Reveals Variants in ARID1A, GPRC5A and MLL2 Driving Self-Renewal of Human Bladder Cancer Stem Cells. Eur Urol (2017) 71:8-12. doi: 10.1016/ j.eururo.2016.06.025

34. Song Q, Su J, Miller LD, Zhang W. scLM: Automatic Detection of Consensus Gene Clusters Across Multiple Single-Cell Datasets. Genomics Proteomics Bioinf (2021) 19:330-41. doi: 10.1016/j.gpb.2020.09.002

35. Song Q, Su J, Zhang W. scGCN Is a Graph Convolutional Networks Algorithm for Knowledge Transfer in Single Cell Omics. Nat Commun (2021) 12:3826. doi: 10.1038/s41467-021-24172-y

36. De P, Aske J, Dey N. Cancer-Associated Fibroblast Functions as a RoadBlock in Cancer Therapy. Cancers (Basel) (2021) 13(20):5246. doi: 10.3390/ cancers 13205246

37. Gunaydin G. CAFs Interacting With TAMs in Tumor Microenvironment to Enhance Tumorigenesis and Immune Evasion. Front Oncol (2021) 11:668349. doi: 10.3389/fonc.2021.668349

38. Nair N, Calle AS, Zahra MH, Prieto-Vila M, Oo AKK, Hurley L, et al. A Cancer Stem Cell Model as the Point of Origin of Cancer-Associated Fibroblasts in Tumor Microenvironment. Sci Rep (2017) 7:6838. doi: 10.1038/s41598-017-07144-5

39. Quante M, Tu SP, Tomita H, Gonda T, Wang SSW, Takashi S, et al. Bone Marrow-Derived Myofibroblasts Contribute to the Mesenchymal Stem Cell Niche and Promote Tumor Growth. Cancer Cell (2011) 19:257-72. doi: 10.1016/j.ccr.2011.01.020

40. Sugimoto H, Mundel TM, Kieran MW, Kalluri R. Identification of Fibroblast Heterogeneity in the Tumor Microenvironment. Cancer Biol Ther (2006) 5:1640-6. doi: 10.4161/cbt.5.12.3354

41. Kobayashi H, Enomoto A, Woods SL, Burt AD, Takahashi M, Worthley DL. Cancer-Associated Fibroblasts in Gastrointestinal Cancer. Nat Rev Gastroenterol Hepatol (2019) 16:282-95. doi: 10.1038/s41575-019-0115-0

42. Hasegawa T, Yashiro M, Nishii T, Matsuoka J, Fuyuhiro Y, Morisaki T, et al. Cancer-Associated Fibroblasts Might Sustain the Stemness of Scirrhous Gastric Cancer Cells via Transforming Growth Factor- $\beta$ Signaling. Int $J$ Cancer (2014) 134:1785-95. doi: 10.1002/ijc.28520

43. Karakasheva TA, Lin EW, Tang Q, Qiao E, Waldron TJ, Soni M, et al. IL-6 Mediates Cross-Talk Between Tumor Cells and Activated Fibroblasts in the Tumor Microenvironment. Cancer Res (2018) 78:4957-70. doi: 10.1158/ 0008-5472.CAN-17-2268

44. Wang X, Zhou Q, Yu Z, Wu X, Chen X, Li J, et al. Cancer-Associated Fibroblast-Derived Lumican Promotes Gastric Cancer Progression via the Integrin $\beta 1$-FAK Signaling Pathway. Int J Cancer (2017) 141:998-1010. doi: $10.1002 / \mathrm{ijc} .30801$ 
45. Shibata W, Ariyama H, Westphalen CB, Worthley DL, Muthupalani S, Asfaha S, et al. Stromal Cell-Derived Factor-1 Overexpression Induces Gastric Dysplasia Through Expansion of Stromal Myofibroblasts and Epithelial Progenitors. Gut (2013) 62:192-200. doi: 10.1136/gutjnl-2011301824

46. Huang L, Xu AM, Liu S, Liu W, Li T-J. Cancer-Associated Fibroblasts in Digestive Tumors. World J Gastroenterol (2014) 20:17804-18. doi: 10.3748/ wjg.v20.i47.17804

47. Bockerstett KA, DiPaolo RJ. Regulation of Gastric Carcinogenesis by Inflammatory Cytokines. Cell Mol Gastroenterol Hepatol (2017) 4:47-53. doi: 10.1016/j.jcmgh.2017.03.005

48. Li P, Shan J-X, Chen X-H, Zhang D, Su L-P, Huang X-Y, et al. Epigenetic Silencing of microRNA-149 in Cancer-Associated Fibroblasts Mediates Prostaglandin E2/interleukin-6 Signaling in the Tumor Microenvironment. Cell Res (2015) 25:588-603. doi: 10.1038/cr.2015.51

49. Kinoshita H, Hirata Y, Nakagawa H, Sakamoto K, Hayakawa Y, Takahashi $\mathrm{R}$, et al. Interleukin-6 Mediates Epithelial-Stromal Interactions and Promotes Gastric Tumorigenesis. PLoS One (2013) 8:e60914. doi: 10.1371/ journal.pone.0060914

50. Gu J, Qian H, Shen L, Zhang X, Zhu W, Huang L, et al. Gastric Cancer Exosomes Trigger Differentiation of Umbilical Cord Derived Mesenchymal Stem Cells to Carcinoma-Associated Fibroblasts Through TGF- $\beta /$ Smad Pathway. PLoS One (2012) 7:e52465. doi: 10.1371/journal.pone.0052465

51. Kuramitsu S, Masuda T, Hu Q, Tobo T, Yashiro M, Fujii A, et al. CancerAssociated Fibroblast-Derived Spondin-2 Promotes Motility of Gastric Cancer Cells. Cancer Genomics Proteomics (2021) 18:521-9. doi: 10.21873/ cgp. 20277

52. Ishimoto T, Miyake K, Nandi T, Yashiro M, Onishi N, Huang KK, et al. Activation of Transforming Growth Factor Beta 1 Signaling in Gastric Cancer-Associated Fibroblasts Increases Their Motility, via Expression of Rhomboid 5 Homolog 2, and Ability to Induce Invasiveness of Gastric Cancer Cells. Gastroenterology (2017) 153:191-204. doi: 10.1053/j.gastro.2017.03.046

53. Wen X, He X, Jiao F, Wang C, Sun Y, Ren X, et al. Fibroblast Activation Protein- $\alpha$-Positive Fibroblasts Promote Gastric Cancer Progression and Resistance to Immune Checkpoint Blockade. Oncol Res (2017) 25:629-40. doi: 10.3727/096504016X14768383625385

54. Zheng H, Liu H, Li H, Dou W, Wang X. Weighted Gene Co-Expression Network Analysis Identifies a Cancer-Associated Fibroblast Signature for Predicting Prognosis and Therapeutic Responses in Gastric Cancer. Front Mol Biosci (2021) 8:744677. doi: 10.3389/fmolb.2021.744677

55. Li Y, Li J-Q, Jiang H-P, Li X. The Upregulation of PLXDC2 Correlates With Immune Microenvironment Characteristics and Predicts Prognosis in Gastric Cancer. Dis Markers (2021) 2021:5669635. doi: 10.1155/2021/5669635

56. Rihawi K, Ricci AD, Rizzo A, Brocchi S, Marasco G, Pastore LV, et al. Tumor-Associated Macrophages and Inflammatory Microenvironment in Gastric Cancer: Novel Translational Implications. Int J Mol Sci (2021) 22 (8):3805. doi: 10.3390/ijms22083805

57. Gambardella V, Castillo J, Tarazona N, Gimeno-Valiente F, MartínezCiarpaglini C, Cabeza-Segura M, et al. The Role of Tumor-Associated Macrophages in Gastric Cancer Development and Their Potential as a Therapeutic Target. Cancer Treat Rev (2020) 86:102015. doi: 10.1016/ j.ctrv.2020.102015

58. Petersen CP, Meyer AR, De Salvo C, Choi E, Schlegel C, Petersen A, et al. A Signalling Cascade of IL-33 to IL-13 Regulates Metaplasia in the Mouse Stomach. Gut (2018) 67:805-17. doi: 10.1136/gutjnl-2016-312779

59. Oishi S, Takano R, Tamura S, Tani S, Iwaizumi M, Hamaya Y, et al. M2 Polarization of Murine Peritoneal Macrophages Induces Regulatory Cytokine Production and Suppresses T-Cell Proliferation. Immunology (2016) 149:320-8. doi: 10.1111/imm.12647

60. Oguma K, Oshima H, Aoki M, Uchio R, Naka K, Nakamura S, et al. Activated Macrophages Promote Wnt Signalling Through Tumour Necrosis Factor-Alpha in Gastric Tumour Cells. EMBO J (2008) 27:1671-81. doi: 10.1038/emboj.2008.105

61. Oshima H, Hioki K, Popivanova BK, Oguma K, Van Rooijen N, Ishikawa T$\mathrm{O}$, et al. Prostaglandin $\mathrm{E}_{2}$ Signaling and Bacterial Infection Recruit TumorPromoting Macrophages to Mouse Gastric Tumors. Gastroenterology (2011) 140:596-607. doi: 10.1053/j.gastro.2010.11.007
62. Yamanaka N, Morisaki T, Nakashima H, Tasaki A, Kubo M, Kuga H, et al. Interleukin 1beta Enhances Invasive Ability of Gastric Carcinoma Through Nuclear factor-kappaB Activation. Clin Cancer Res (2004) 10:1853-9. doi: 10.1158/1078-0432.CCR-03-0300

63. Ohta M, Kitadai Y, Tanaka S, Yoshihara M, Yasui W, Mukaida N, et al. Monocyte Chemoattractant Protein-1 Expression Correlates With Macrophage Infiltration and Tumor Vascularity in Human Gastric Carcinomas. Int J Oncol (2003) 22:773-8. doi: 10.3892/ijo.22.4.773

64. Kuroda T, Kitadai Y, Tanaka S, Yang X, Mukaida N, Yoshihara M, et al. Monocyte Chemoattractant Protein-1 Transfection Induces Angiogenesis and Tumorigenesis of Gastric Carcinoma in Nude Mice via Macrophage Recruitment. Clin Cancer Res (2005) 11:7629-36. doi: 10.1158/1078-0432.CCR-05-0798

65. Wu H, Xu J-B, He Y-L, Peng J-J, Zhang X-H, Chen C-Q, et al. TumorAssociated Macrophages Promote Angiogenesis and Lymphangiogenesis of Gastric Cancer. J Surg Oncol (2012) 106:462-8. doi: 10.1002/jso.23110

66. Jiménez $G$, Hackenberg $M$, Catalina $P$, Boulaiz H, Griñán-Lisón C, García MÁ, et al. Mesenchymal Stem Cell's Secretome Promotes Selective Enrichment of Cancer Stem-Like Cells With Specific Cytogenetic Profile. Cancer Lett (2018) 429:78-88. doi: 10.1016/j.canlet.2018.04.042

67. Li W, Zhou Y, Yang J, Zhang X, Zhang H, Zhang T, et al. Gastric CancerDerived Mesenchymal Stem Cells Prompt Gastric Cancer Progression Through Secretion of Interleukin-8. J Exp Clin Cancer Res (2015) 34:52. doi: 10.1186/s13046-015-0172-3

68. Sun L, Wang Q, Chen B, Zhao Y, Shen B, Wang X, et al. Human Gastric Cancer Mesenchymal Stem Cell-Derived IL15 Contributes to Tumor Cell Epithelial-Mesenchymal Transition via Upregulation Tregs Ratio and PD-1 Expression in CD4T Cell. Stem Cells Dev (2018) 27:1203-14. doi: 10.1089/ scd.2018.0043

69. Huang F, Wang M, Yang T, Cai J, Zhang Q, Sun Z, et al. Gastric CancerDerived MSC-Secreted PDGF-DD Promotes Gastric Cancer Progression. J Cancer Res Clin Oncol (2014) 140:1835-48. doi: 10.1007/s00432-014-1723-2

70. Wang M, Zhao C, Shi H, Zhang B, Zhang L, Zhang X, et al. Deregulated microRNAs in Gastric Cancer Tissue-Derived Mesenchymal Stem Cells: Novel Biomarkers and a Mechanism for Gastric Cancer. Br J Cancer (2014) 110:1199-210. doi: 10.1038/bjc.2014.14

71. Li W, Zhang X, Wu F, Zhou Y, Bao Z, Li H, et al. Gastric Cancer-Derived Mesenchymal Stromal Cells Trigger M2 Macrophage Polarization That Promotes Metastasis and EMT in Gastric Cancer. Cell Death Dis (2019) 10:918. doi: 10.1038/s41419-019-2131-y

72. Yin L, Zhang R, Hu Y, Li W, Wang M, Liang Z, et al. Gastric-Cancer-Derived Mesenchymal Stem Cells: A Promising Target for Resveratrol in the Suppression of Gastric Cancer Metastasis. Hum Cell (2020) 33:652-62. doi: $10.1007 /$ s13577-020-00339-5

73. Ikeda T, Nishita M, Hoshi K, Honda T, Kakeji Y, Minami Y. Mesenchymal Stem Cell-Derived CXCL16 Promotes Progression of Gastric Cancer Cells by STAT3-Mediated Expression of Ror1. Cancer Sci (2020) 111:1254-65. doi: $10.1111 /$ cas.14339

74. Takiguchi G, Nishita M, Kurita K, Kakeji Y, Minami Y. Wnt5a-Ror2 Signaling in Mesenchymal Stem Cells Promotes Proliferation of Gastric Cancer Cells by Activating CXCL16-CXCR6 Axis. Cancer Sci (2016) 107:290-7. doi: 10.1111/cas.12871

75. Han J, Khatwani N, Searles TG, Turk MJ, Angeles CV. Memory CD8 T Cell Responses to Cancer. Semin Immunol (2020) 49:101435. doi: 10.1016/ j.smim.2020.101435

76. Liu X, Zhang Z, Zhao G. Recent Advances in the Study of Regulatory T Cells in Gastric Cancer. Int Immunopharmacol (2019) 73:560-7. doi: 10.1016/ j.intimp.2019.05.009

77. Kono K, Kawaida H, Takahashi A, Sugai H, Mimura K, Miyagawa N, et al. CD4(+)CD25high Regulatory T Cells Increase With Tumor Stage in Patients With Gastric and Esophageal Cancers. Cancer Immunol Immunother (2006) 55:1064-71. doi: 10.1007/s00262-005-0092-8

78. Zhuang Y, Peng L-S, Zhao Y-L, Shi Y, Mao X-H, Guo G, et al. Increased Intratumoral IL-22-Producing CD4(+) T Cells and Th22 Cells Correlate With Gastric Cancer Progression and Predict Poor Patient Survival. Cancer Immunol Immunother (2012) 61:1965-75. doi: 10.1007/s00262-012-1241-5

79. Zhuang Y, Peng L-S, Zhao Y-L, Shi Y, Mao X-H, Chen W, et al. CD8(+) T Cells That Produce Interleukin-17 Regulate Myeloid-Derived Suppressor 
Cells and Are Associated With Survival Time of Patients With Gastric Cancer. Gastroenterology (2012) 143:951-62. doi: 10.1053/j.gastro.2012.06.010

80. Sitarz R, Skierucha M, Mielko J, Offerhaus GJA, Maciejewski R, Polkowski WP. Gastric Cancer: Epidemiology, Prevention, Classification, and Treatment. Cancer Manage Res (2018) 10:239-48. doi: 10.2147/CMAR.S149619

81. Amieva M, Peek RM. Pathobiology of Helicobacter Pylori-Induced Gastric Cancer. Gastroenterology (2016) 150:64-78. doi: 10.1053/j.gastro.2015.09.004

82. Baj J, Brzozowska K, Forma A, Maani A, Sitarz E, Portincasa P. Immunological Aspects of the Tumor Microenvironment and EpithelialMesenchymal Transition in Gastric Carcinogenesis. Int J Mol Sci (2020) 21 (7):2544. doi: 10.3390/ijms21072544

83. Soundararajan R, Fradette JJ, Konen JM, Moulder S, Zhang X, Gibbons DL, et al. Targeting the Interplay Between Epithelial-To-MesenchymalTransition and the Immune System for Effective Immunotherapy. Cancers (Basel) (2019) 11(5):714. doi: 10.3390/cancers11050714

84. Ma H-Y, Liu X-Z, Liang C-M. Inflammatory Microenvironment Contributes to Epithelial-Mesenchymal Transition in Gastric Cancer. World J Gastroenterol (2016) 22:6619-28. doi: 10.3748/wjg.v22.i29.6619

85. Krzysiek-Maczka G, Targosz A, Szczyrk U, Strzalka M, Brzozowski T, PtakBelowska A. Involvement of Epithelial-Mesenchymal Transition-Inducing Transcription Factors in the Mechanism of Helicobacter Pylori-Induced Fibroblasts Activation. J Physiol Pharmacol (2019) 70:727-36. doi: 10.26402/ jpp.2019.5.08

86. Zhang Q, Wang M, Huang F, Yang T, Cai J, Zhang X, et al. H. Pylori Infection-Induced MSC Differentiation Into CAFs Promotes EpithelialMesenchymal Transition in Gastric Epithelial Cells. Int J Mol Med (2013) 32:1465-73. doi: 10.3892/ijmm.2013.1532

87. Krzysiek-Maczka G, Targosz A, Szczyrk U, Strzałka M, Sliwowski Z, Brzozowski T, et al. Role of Helicobacter Pylori Infection in CancerAssociated Fibroblast-Induced Epithelial-Mesenchymal Transition In Vitro. Helicobacter (2018) 23:e12538. doi: 10.1111/hel.12538

88. Zhang X, Ashcraft K, Betof Warner A, Nair S, Dewhirst M. Can ExerciseInduced Modulation of the Tumor Physiologic Microenvironment Improve Antitumor Immunity? Cancer Res (2019) 79:2447-56. doi: 10.1158/00085472.Can-18-2468

89. Varisli L, Cen O, Vlahopoulos S. Dissecting Pharmacological Effects of Chloroquine in Cancer Treatment: Interference With Inflammatory Signaling Pathways. Immunology (2020) 159:257-78. doi: 10.1111/ imm. 13160

90. Lai Y, Huang H, Abudoureyimu M, Lin X, Tian C, Wang T, et al. NonCoding RNAs: Emerging Regulators of Glucose Metabolism in Hepatocellular Carcinoma. Am J Cancer Res (2020) 10:4066-84.

91. Yadav UP, Singh T, Kumar P, Sharma P, Kaur H, Sharma S, et al. Metabolic Adaptations in Cancer Stem Cells. Front Oncol (2020) 10:1010. doi: 10.3389/ fonc. 2020.01010

92. Nimmakayala RK, Leon F, Rachagani S, Rauth S, Nallasamy P, Marimuthu S, et al. Metabolic Programming of Distinct Cancer Stem Cells Promotes Metastasis of Pancreatic Ductal Adenocarcinoma. Oncogene (2021) 40:21531. doi: 10.1038/s41388-020-01518-2

93. Hu P-S, Li T, Lin J-F, Qiu M-Z, Wang D-S, Liu Z-X, et al. VDR-SOX2 Signaling Promotes Colorectal Cancer Stemness and Malignancy in an Acidic Microenvironment. Signal transduction targeted Ther (2020) 5:183. doi: 10.1038/s41392-020-00230-7

94. Wojtkowiak JW, Gillies RJ. Autophagy on Acid. Autophagy (2012) 8:1688-9. doi: 10.4161/auto.21501

95. Chen X, Sun X, Wang Z, Zhou X, Xu L, Fe Li, et al. Involvement of AcidSensing Ion Channel la in Gastric Carcinoma Cell Migration and Invasion. Acta Biochim Biophys Sin (2018) 50:440-6. doi: 10.1093/abbs/gmy026

96. Chen L, Kasai T, Li Y, Sugii Y, Jin G, Okada M, et al. A Model of Cancer Stem Cells Derived From Mouse Induced Pluripotent Stem Cells. PLoS One (2012) 7:e33544. doi: 10.1371/journal.pone.0033544

97. Nguyen LV, Vanner R, Dirks P, Eaves CJ. Cancer Stem Cells: An Evolving Concept. Nat Rev Cancer (2012) 12:133-43. doi: 10.1038/nrc3184

98. Nassar D, Blanpain C. Cancer Stem Cells: Basic Concepts and Therapeutic Implications. Annu Rev Pathol (2016) 11:47-76. doi: 10.1146/annurevpathol-012615-044438
99. Najafi M, Farhood B, Mortezaee K. Cancer Stem Cells (CSCs) in Cancer Progression and Therapy. J Cell Physiol (2019) 234:8381-95. doi: 10.1002/ jcp. 27740

100. Huang T, Song X, Xu D, Tiek D, Goenka A, Wu B, et al. Stem Cell Programs in Cancer Initiation, Progression, and Therapy Resistance. Theranostics (2020) 10:8721-43. doi: 10.7150/thno.41648

101. Dzobo K, Senthebane DA, Ganz C, Thomford NE, Wonkam A, Dandara C. Advances in Therapeutic Targeting of Cancer Stem Cells Within the Tumor Microenvironment: An Updated Review. Cells (2020) 9(8):1896. doi: $10.3390 /$ cells 9081896

102. Borovski T, De Sousa E Melo F, Vermeulen L, Medema J. Cancer Stem Cell Niche: The Place to Be. Cancer Res (2011) 71:634-9. doi: 10.1158/00085472. Can-10-3220

103. Sottoriva A, Sloot PMA, Medema JP, Vermeulen L. Exploring Cancer Stem Cell Niche Directed Tumor Growth. Cell Cycle (Georgetown Tex.) (2010) 9:1472-9. doi: 10.4161/cc.9.8.11198

104. Zhang J, Gao B, Zhang W, Qian Z, Xiang Y. Monitoring Antiangiogenesis of Bevacizumab in Zebrafish. Drug Design Dev Ther (2018) 12:2423-30. doi: 10.2147/DDDT.S166330

105. Lv Y, Song L, Chang L, Zhang X, Liu Y, Liu W. Effect of Bevacizumab Combined With Chemotherapy at Different Sequences in the GastricCancer-Bearing Nude Mice. J Cancer Res Ther (2018) 14:S190-6. doi: 10.4103/0973-1482.171364

106. Ma J, Yao S, Li X-S, Kang H-R, Yao F-F, Du N. Neoadjuvant Therapy of DOF Regimen Plus Bevacizumab Can Increase Surgical Resection Ratein Locally Advanced Gastric Cancer: A Randomized, Controlled Study. Medicine (2015) 94:e1489. doi: 10.1097/MD.0000000000001489

107. Singh R, Kim WJ, Kim P-H, Hong HJ. Combined Blockade of HER2 and VEGF Exerts Greater Growth Inhibition of HER2-Overexpressing Gastric Cancer Xenografts Than Individual Blockade. Exp Mol Med (2013) 45:e52. doi: $10.1038 / \mathrm{emm} .2013 .111$

108. Das B, Tsuchida R, Malkin D, Koren G, Baruchel S, Yeger H. Hypoxia Enhances Tumor Stemness by Increasing the Invasive and Tumorigenic Side Population Fraction. Stem Cells (2008) 26:1818-30. doi: 10.1634/ stemcells.2007-0724

109. Pistollato F, Rampazzo E, Persano L, Abbadi S, Frasson C, Denaro L, et al. Interaction of Hypoxia-Inducible Factor- $1 \alpha$ and Notch Signaling Regulates Medulloblastoma Precursor Proliferation and Fate. Stem Cells (2010) 28:1918-29. doi: 10.1002/stem.518

110. Scheel C, Eaton EN, Li SH-J, Chaffer CL, Reinhardt F, Kah K-J, et al. Paracrine and Autocrine Signals Induce and Maintain Mesenchymal and Stem Cell States in the Breast. Cell (2011) 145:926-40. doi: 10.1016/ j.cell.2011.04.029

111. Man J, Yu X, Huang H, Zhou W, Xiang C, Huang H, et al. Hypoxic Induction of Vasorin Regulates Notch1 Turnover to Maintain Glioma Stem-Like Cells. Cell Stem Cell (2018) 22:104-18. doi: 10.1016/j.stem.2017.10.005

112. Maeda M, Takeshima H, Iida N, Hattori N, Yamashita S, Moro H, et al. Cancer Cell Niche Factors Secreted From Cancer-Associated Fibroblast by Loss of H3k27me3. Gut (2020) 69:243-51. doi: 10.1136/gutjnl-2018-317645

113. Kato Y, Yashiro M, Fuyuhiro Y, Kashiwagi S, Matsuoka J, Hirakawa T, et al. Effects of Acute and Chronic Hypoxia on the Radiosensitivity of Gastric and Esophageal Cancer Cells. Anticancer Res (2011) 31:3369-75.

114. Liu L, Ning X, Sun L, Zhang H, Shi Y, Guo C, et al. Hypoxia-Inducible Factor-1 Alpha Contributes to Hypoxia-Induced Chemoresistance in Gastric Cancer. Cancer Sci (2008) 99:121-8. doi: 10.1111/j.1349-7006.2007.00643.x

115. Matsuoka J, Yashiro M, Doi Y, Fuyuhiro Y, Kato Y, Shinto O, et al. Hypoxia Stimulates the EMT of Gastric Cancer Cells Through Autocrine Tgf $\beta$ Signaling. PLoS One (2013) 8:e62310. doi: 10.1371/journal.pone.0062310

116. Pardoll DM. The Blockade of Immune Checkpoints in Cancer Immunotherapy. Nat Rev Cancer (2012) 12:252-64. doi: 10.1038/nrc3239

117. Wu X, Gu Z, Chen Y, Chen B, Chen W, Weng L, et al. Application of PD-1 Blockade in Cancer Immunotherapy. Comput Struct Biotechnol J (2019) 17:661-74. doi: 10.1016/j.csbj.2019.03.006

118. Sundar R, Smyth E, Peng S, Yeong J, Tan P. Predictive Biomarkers of Immune Checkpoint Inhibition in Gastroesophageal Cancers. Front Oncol (2020) 10:763. doi: 10.3389/fonc.2020.00763 
119. Wang Z-F, Ma D-G, Zhu Z, Mu Y-P, Yang Y-Y, Feng L, et al. Astragaloside IV Inhibits Pathological Functions of Gastric Cancer-Associated Fibroblasts. World J Gastroenterol (2017) 23:8512-25. doi: 10.3748/wjg.v23.i48.8512

120. Zhang H, Deng T, Liu R, Ning T, Yang H, Liu D, et al. CAF Secreted miR-522 Suppresses Ferroptosis and Promotes Acquired Chemo-Resistance in Gastric Cancer. Mol Cancer (2020) 19:43. doi: 10.1186/s12943-020-01168-8

121. Uchihara T, Miyake K, Yonemura A, Komohara Y, Itoyama R, Koiwa M, et al. Extracellular Vesicles From Cancer-Associated Fibroblasts Containing Annexin A6 Induces FAK-YAP Activation by Stabilizing $\beta 1$ Integrin, Enhancing Drug Resistance. Cancer Res (2020) 80:3222-35. doi: 10.1158/ 0008-5472.CAN-19-3803

122. Shen J, Zhai J, You Q, Zhang G, He M, Yao X, et al. Cancer-Associated Fibroblasts-Derived VCAM1 Induced by $\mathrm{H}$. Pylori Infection Facilitates Tumor Invasion in Gastric Cancer. Oncogene (2020) 39:2961-74. doi: 10.1038/s41388-020-1197-4

123. Miao L, Qi J, Zhao Q, Wu Q-N, Wei D-L, Wei X-L, et al. Targeting the STING Pathway in Tumor-Associated Macrophages Regulates Innate Immune Sensing of Gastric Cancer Cells. Theranostics (2020) 10:498-515. doi: 10.7150/thno.37745

124. Zheng P, Chen L, Yuan X, Luo Q, Liu Y, Xie G, et al. Exosomal Transfer of Tumor-Associated Macrophage-Derived miR-21 Confers Cisplatin Resistance in Gastric Cancer Cells. J Exp Clin Cancer Res (2017) 36:53. doi: 10.1186/s13046-017-0528-y

125. Wang H, Chen B, Lin Y, Zhou Y, Li X. Legumain Promotes Gastric Cancer Progression Through Tumor-Associated Macrophages and. Int J Biol Sci (2020) 16:172-80. doi: 10.7150/ijbs.36467

126. Sun L, Huang C, Zhu M, Guo S, Gao Q, Wang Q, et al. Gastric Cancer Mesenchymal Stem Cells Regulate PD-L1-CTCF Enhancing Cancer Stem Cell-Like Properties and Tumorigenesis. Theranostics (2020) 10:11950-62. doi: 10.7150/thno.49717

127. He W, Liang B, Wang C, Li S, Zhao Y, Huang Q, et al. MSC-Regulated lncRNA MACC1-AS1 Promotes Stemness and Chemoresistance Through Fatty Acid Oxidation in Gastric Cancer. Oncogene (2019) 38:4637-54. doi: 10.1038/s41388-019-0747-0

128. Ji R, Zhang B, Zhang X, Xue J, Yuan X, Yan Y, et al. Exosomes Derived From Human Mesenchymal Stem Cells Confer Drug Resistance in Gastric Cancer. Cell Cycle (Georgetown Tex.) (2015) 14:2473-83. doi: 10.1080/15384101.2015.1005530

129. Yao H-J, Zhang Y-G, Sun L, Liu Y. The Effect of Hyaluronic Acid Functionalized Carbon Nanotubes Loaded With Salinomycin on Gastric Cancer Stem Cells. Biomaterials (2014) 35:9208-23. doi: 10.1016/ j.biomaterials.2014.07.033
130. Liang S, Li C, Zhang C, Chen Y, Xu L, Bao C, et al. CD44v6 Monoclonal Antibody-Conjugated Gold Nanostars for Targeted Photoacoustic Imaging and Plasmonic Photothermal Therapy of Gastric Cancer Stem-Like Cells. Theranostics (2015) 5:970-84. doi: 10.7150/thno.11632

131. Barat S, Chen X, Cuong Bui K, Bozko P, Götze J, Christgen M, et al. GammaSecretase Inhibitor IX (GSI) Impairs Concomitant Activation of Notch and Wnt-Beta-Catenin Pathways in CD44 Gastric Cancer Stem Cells. Stem Cells Trans Med (2017) 6:819-29. doi: 10.1002/sctm.16-0335

132. Feng S, Zheng Z, Feng L, Yang L, Chen Z, Lin Y, et al. Proton Pump Inhibitor Pantoprazole Inhibits the Proliferation, Self-Renewal and Chemoresistance of Gastric Cancer Stem Cells via the EMT/ $\beta$-Catenin Pathways. Oncol Rep (2016) 36:3207-14. doi: 10.3892/or.2016.5154

133. Yoon C, Park DJ, Schmidt B, Thomas NJ, Lee H-J, Kim TS, et al. CD44 Expression Denotes a Subpopulation of Gastric Cancer Cells in Which Hedgehog Signaling Promotes Chemotherapy Resistance. Clin Cancer Res (2014) 20:3974-88. doi: 10.1158/1078-0432.CCR-14-0011

134. Mao J, Fan S, Ma W, Fan P, Wang B, Zhang J, et al. Roles of Wnt/B-Catenin Signaling in the Gastric Cancer Stem Cells Proliferation and Salinomycin Treatment. Cell Death Dis (2014) 5:e1039. doi: 10.1038/cddis.2013.515

135. Wang B, Liu J, Ma LN, Xiao HL, Wang YZ, Li Y, et al. Chimeric 5/35 Adenovirus-Mediated Dickkopf-1 Overexpression Suppressed Tumorigenicity of CD $44^{+}$Gastric Cancer Cells via Attenuating Wnt Signaling. J Gastroenterol (2013) 48:798-808. doi: 10.1007/s00535-012-0711-z

Conflict of Interest: The authors declare that the research was conducted in the absence of any commercial or financial relationships that could be construed as a potential conflict of interest.

Publisher's Note: All claims expressed in this article are solely those of the authors and do not necessarily represent those of their affiliated organizations, or those of the publisher, the editors and the reviewers. Any product that may be evaluated in this article, or claim that may be made by its manufacturer, is not guaranteed or endorsed by the publisher.

Copyright (C) 2022 Yang, Meng and Wang. This is an open-access article distributed under the terms of the Creative Commons Attribution License (CC BY). The use, distribution or reproduction in other forums is permitted, provided the original author(s) and the copyright owner(s) are credited and that the original publication in this journal is cited, in accordance with accepted academic practice. No use, distribution or reproduction is permitted which does not comply with these terms. 